\title{
Improving the Crossing Lemma by Finding More Crossings in Sparse Graphs*
}

\author{
János Pach, ${ }^{1,2}$ Radoš Radoičić, ${ }^{3}$ Gábor Tardos, ${ }^{2}$ and Géza Tóth ${ }^{2}$ \\ ${ }^{1}$ Courant Institute, N.Y.U., \\ 251 Mercer Street, New York, NY 10012, USA \\ pach@cs.ccny.cuny.edu \\ ${ }^{2}$ Rényi Institute of Mathematics, Hungarian Academy of Sciences, \\ Pf. 127, H-1364 Budapest, Hungary \\ \{tardos,toth\}@ renyi.hu \\ ${ }^{3}$ Department of Mathematics, Baruch College, CUNY, \\ One Bernard Baruch Way, New York, NY 10010, USA \\ radoicic@baruch.cuny.edu
}

\begin{abstract}
Twenty years ago, Ajtai et al. and, independently, Leighton discovered that the crossing number of any graph with $v$ vertices and $e>4 v$ edges is at least $c e^{3} / v^{2}$, where $c>0$ is an absolute constant. This result, known as the "Crossing Lemma," has found many important applications in discrete and computational geometry. It is tight up to a multiplicative constant. Here we improve the best known value of the constant by showing that the result holds with $c>1024 / 31827>0.032$. The proof has two new ingredients, interesting in their own right. We show that (1) if a graph can be drawn in the plane so that every edge crosses at most three others, then its number of edges cannot exceed 5.5 $(v-2)$; and (2) the crossing number of any graph is at least $\frac{7}{3} e-\frac{25}{3}(v-2)$. Both bounds are tight up to an additive constant (the latter one in the range $4 v \leq e \leq 5 v$ ).
\end{abstract}

\section{Introduction}

Unless stated otherwise, the graphs considered in this paper have no loops or parallel edges. The number of vertices and number of edges of a graph $G$ are denoted by $v(G)$ and $e(G)$, respectively. We say that $G$ is drawn in the plane if its vertices are represented

* J. Pach was supported by NSF Grant CCR-00-98246, PSC-CUNY Research Award 63382-0032, and OTKA T-032452. R. Radoičić was supported by Central European University, Budapest, and G. Tóth was supported by OTKA T-038397. 
by distinct points and its edges by (possibly intersecting) Jordan arcs connecting the corresponding point pairs. If it leads to no confusion, in terminology and notation we make no distinction between the vertices of $G$ and the corresponding points, or between the edges and the corresponding Jordan arcs. We always assume that in a drawing (a) no edge passes through a vertex different from its endpoints, (b) no three edges cross at the same point, and (c) any two edges have only a finite number of interior points in common, and at these points they properly cross, i.e., one of the edges passes from one side of the other edge to the other side (see [P1] and [P2]). A crossing between two edges is their common interior point (if it exists). The crossing number of $G$, denoted by $\operatorname{cr}(G)$, is the minimum number of crossings in a drawing of $G$ satisfying the above conditions.

Ajtai et al. $\left[\mathrm{AC}^{+}\right]$and, independently, Leighton [L] have proved the following result, which is usually referred to as the "Crossing Lemma." The crossing number of any graph with $v$ vertices and $e>4 v$ edges satisfies

$$
\operatorname{cr}(G) \geq \frac{1}{64} \frac{e^{3}}{v^{2}} .
$$

This result, which is tight apart from the value of the constant, has found many applications in combinatorial geometry, convexity, number theory, and VLSI design (see [L], [Sz], [PS], [ENR], [STT], and [PTa]). In particular, it has played a pivotal role in obtaining the best known upper bound on the number of $k$-sets [D] and lower bound on the number of distinct distances determined by $n$ points in the plane [ST], [KT]. According to a conjecture of Erdős and Guy [ErG], which was verified in [PST], as long as $e / v \rightarrow \infty$ and $e / v^{2} \rightarrow 0$, the limit

$$
\lim _{v \rightarrow \infty} \min _{\substack{v(G)=v \\ e(G)=e}} \frac{\operatorname{cr}(G)}{e^{3} / v^{2}}
$$

exists. The best known upper and lower bounds for this constant (roughly 0.09 and $1 / 33.75 \approx 0.029$, resp.) were obtained in [PTo1].

All known proofs of the Crossing Lemma are based on the trivial inequality $\operatorname{cr}(H) \geq$ $e(H)-(3 v(H)-6)$, which is an immediate corollary of Euler's Polyhedral Formula $(v(H)>2)$. Applying this statement inductively to all small (and mostly sparse) subgraphs $H \subseteq G$ or to a randomly selected one, the lemma follows. The main idea in [PTo1] was to obtain stronger inequalities for the sparse subgraphs $H$, which have led to better lower bounds on the crossing numbers of all graphs $G$. In this paper we follow the same approach.

For $k \geq 0$, let $e_{k}(v)$ denote the maximum number of edges in a graph of $v \geq 2$ vertices that can be drawn in the plane so that every edge is involved in at most $k$ crossings. By Euler's Formula, we have $e_{0}(v)=3(v-2)$. Pach and Tóth [PTo1] proved that $e_{k}(v) \leq(k+3)(v-2)$, for $0 \leq k \leq 3$. Moreover, for $0 \leq k \leq 2$, these bounds are tight for infinitely many values of $v$. However, for $k=3$, there was a gap between the lower and upper estimates. Our first theorem, whose proof is presented in Section 2, fills this gap. 
Theorem 1. Let $G$ be a graph on $v \geq 3$ vertices that can be drawn in the plane so that each of its edges crosses at most three others. Then we have

$$
e(G) \leq 5.5(v-2) .
$$

Consequently, the maximum number of edges over all such graphs satisfies $e_{3}(v) \leq$ $5.5(v-2)$, and this bound is tight up to an additive constant.

As we have pointed out before, the inequality $e_{0}(v) \leq 3(v-2)$ immediately implies that if a graph $G$ of $v$ vertices has more than $3(v-2)$ edges, then every edge beyond this threshold contributes at least one to $\operatorname{cr}(G)$. Similarly, it follows from inequality $e_{1}(v) \leq 4(v-2)$ that, if $e(G) \geq 4(v-2)$, then every edge beyond 4(v-2) must contribute an additional crossing to $\operatorname{cr}(G)$ (i.e., altogether at least two crossings). Summarizing, we obtain that

$$
\operatorname{cr}(G) \geq(e(G)-3(v(G)-2))+(e(G)-4(v(G)-2)) \geq 2 e(G)-7(v(G)-2)
$$

holds for every graph $G$. Both components of this inequality are tight, so one might expect that their combination cannot be improved either, at least in the range when $e(G)$ is not much larger that $4(v-2)$. However, this is not the case, as is shown by our next result, proved in Section 3.

Theorem 2. The crossing number of any graph $G$ with $v(G) \geq 3$ vertices and $e(G)$ edges satisfies

$$
\operatorname{cr}(G) \geq \frac{7}{3} e(G)-\frac{25}{3}(v(G)-2) .
$$

This bound is tight up to an additive constant whenever $4(v(G)-2) \leq e(G) \leq 5(v(G)-$ 2).

As an application of the above two theorems, in Section 4 we establish the following improved version of the Crossing Lemma.

Theorem 3. The crossing number of any graph $G$ satisfies

$$
\operatorname{cr}(G) \geq \frac{1}{31.1} \frac{e^{3}(G)}{v^{2}(G)}-1.06 v(G) .
$$

If $e(G) \geq \frac{103}{16} v(G)$, we also have

$$
\operatorname{cr}(G) \geq \frac{1024}{31827} \frac{e^{3}(G)}{v^{2}(G)} .
$$

Note for comparison that $1024 / 31827 \approx 1 / 31.08 \approx 0.032$.

In the last section, we adapt the ideas of Székely [Sz] to deduce some consequences of Theorem 3, including an improved version of the Szemerédi-Trotter theorem [SzT] on the maximum number of incidences between $n$ points and $m$ lines. We also discuss some open problems and make a few conjectures and concluding remarks. 
All drawings considered in this paper satisfy the condition that any pair of edges have at most one point in common. This may be either an endpoint or a proper crossing. It is well known and easy to see that every drawing of a graph $G$ that minimizes the number of crossings meets this requirement. Thus, in the proofs of Theorems 2 and 3 , we can make this assumption without loss of generality. However, it is not so obvious whether the same restriction can be justified in the case of Theorem 1. Indeed, in [PTo1], the bound $e(G) \leq(k+3)(v(G)-2)$ was proved only for graphs that can be drawn with at most $k \leq 4$ crossings per edge and which satisfy this extra condition. To prove Theorem 1 in its full generality, we have to establish the following simple statement.

Lemma 1.1. Let $k \leq 3$, and let $G$ be a graph of $v$ vertices that can be drawn in the plane so that each of its edges participates in at most $k$ crossings. In any drawing with this property that minimizes the total number of crossings, every pair of edges has at most one point in common.

Proof. Suppose for contradiction that some pair of edges, $e$ and $f$, have at least two points in common, $A$ and $B$. At least one of these points, say $B$, must be a proper crossing. First, try to swap the portions of $e$ and $f$ between $A$ and $B$, and modify the new drawing in small neighborhoods of $A$ and $B$ so as to reduce the number of crossings between the two edges. Clearly, during this process the number of crossings along any other edge distinct from $e$ and $f$ remains unchanged. The only possible problem that may arise is that after the operation either $e$ or $f$ (say $e$ ) will participate in more than $k$ crossings. In this case, before the operation there were at least two more crossings inside the portion of $f$ between $A$ and $B$, than inside the portion of $e$ between $A$ and $B$. Since $f$ participated in at most three crossings (at most two, not counting $B$ ), we conclude that in the original drawing the portion of $e$ between $A$ and $B$ contained no crossing. If this is the case, instead of swapping the two portions, replace the portion of $f$ between $A$ and $B$ by an arc that runs very close to the portion of $e$ between $A$ and $B$, without intersecting it.

It is interesting to note that the above argument fails for $k \geq 4$, as shown in Fig. 1.

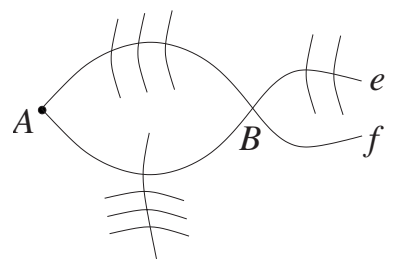

Fig. 1. Two adjacent edges $e$ and $f$ cross, each participating in exactly four crossings. 


\section{Proof of Theorem 1}

We use induction on $v$. For $v \leq 4$, the statement is trivial. Let $v>4$, and suppose that the theorem has already been proved for graphs having fewer than $v$ vertices.

Let $\mathcal{G}$ denote the set of all triples $\left(G, G^{\prime}, \mathcal{D}\right)$ where $G$ is a graph of $v$ vertices, $\mathcal{D}$ is a drawing of $G$ in the plane such that every edge of $G$ crosses at most three others (and every pair of edges has at most one point in common), and $G^{\prime}$ is a planar subgraph of $G$ with $V\left(G^{\prime}\right)=V(G)$ that satisfies the condition that no two arcs in $\mathcal{D}$ representing edges of $G^{\prime}$ cross each other. Let $\mathcal{G}^{\prime} \subset \mathcal{G}$ consist of all elements $\left(G, G^{\prime}, \mathcal{D}\right) \in \mathcal{G}$ for which the number of edges of $G$ is maximum. Finally, let $\mathcal{G}^{\prime \prime} \subset \mathcal{G}^{\prime}$ consist of all elements of $\mathcal{G}^{\prime}$ for which the number of edges of $G^{\prime}$ is maximum. Fix a triple $\left(G, G^{\prime}, \mathcal{D}\right) \in \mathcal{G}^{\prime \prime}$ such that the total number of crossings in $\mathcal{D}$ along all edges of $G^{\prime}$ is as small as possible. This triple remains fixed throughout the whole argument. The term face, unless explicitly stated otherwise, refers to a face of the planar drawing of $G^{\prime}$ induced by $\mathcal{D}$. For any face $\Phi$ (of $G^{\prime}$ ), let $|\Phi|$ denote its number of sides, i.e., the number of edges of $G^{\prime}$ along the boundary of $\Phi$, where every edge whose both sides belong to the interior of $\Phi$ is counted twice. Notice that $|\Phi| \geq 3$ for every face $\Phi$, unless $G^{\prime}$ consists of a single edge, in which case $v(G) \leq 4$, a contradiction.

It follows from the maximality of $G^{\prime}$ that every edge $e$ of $G$ that does not belong to $G^{\prime}$ (in short, $e \in G-G^{\prime}$ ) crosses at least one edge of $G^{\prime}$. The closed portion between an endpoint of $e$ and the nearest crossing of $e$ with an edge of $G^{\prime}$ is called a half-edge. We orient every half-edge from its endpoint which is a vertex of $G$ (and $G^{\prime}$ ) towards its other end sitting in the interior of an edge of $G^{\prime}$. Clearly, every edge $e \in G-G^{\prime}$ has two oriented half-edges. Every half-edge lies in a face $\Phi$ and contains at most two crossings with edges of $G$ in its interior. The extension of a half-edge is the edge of $G-G^{\prime}$ it belongs to. The set of half-edges belonging to a face $\Phi$ is denoted by $H(\Phi)$.

Lemma 2.1. Let $\Phi$ be a face of $G^{\prime}$, and let $g$ be one of its sides. Then $H(\Phi)$ cannot contain two non-crossing half-edges, both of which end on $g$ and cross two other edges of $G$ (that are not necessarily the same).

Proof. Let $e_{1}$ and $e_{2}$ denote the extensions of two non-crossing half-edges in $\Phi$ that end on $g$. Both half-edges cross two edges of $G$, so their extensions cannot cross any other edge apart from $g$. Removing $g$ from $G^{\prime}$ and adding $e_{1}$ and $e_{2}$, we would obtain a larger plane subgraph of $G$, contradicting the maximality of $G^{\prime}$ (Fig. 2).

A face $\Phi$ of $G^{\prime}$ is called simple if its boundary is connected and it does not contain any isolated vertex of $G^{\prime}$ in its interior.

Lemma 2.2. The number of half-edges in any simple face $\Phi$ satisfies

$$
|H(\Phi)| \leq 3|\Phi|-6 .
$$

Proof. For an induction argument to go through, it will be more convenient to prove the lemma for more general configurations. Slightly abusing the terminology and the 

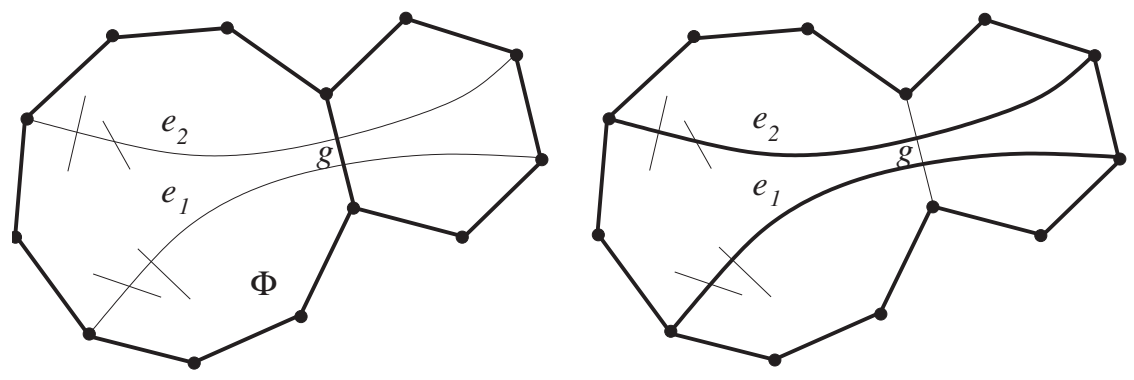

Fig. 2. Lemma 2.1; the edges of $G^{\prime}$ are drawn in bold.

notation, we prove the inequality $|H(\Phi)| \leq 3|\Phi|-6$, for any simple "face" $\Phi$ with $|\Phi| \geq 3$ ( $\Phi$ may have nothing to do with $G$ or $G^{\prime}$ ) and for any set of oriented "halfedges" $H(\Phi)$ contained in $\Phi$ that satisfy the following conditions:

(i) Every half-edge in $H(\Phi)$ emanates from a vertex of $\Phi$ and ends at an edge of $\Phi$ not incident to that vertex.

(ii) The number of half-edges ending at any edge of $\Phi$ is at most three.

(iii) Every half-edge belonging to $H(\Phi)$ crosses at most two others.

(iv) If there are two non-crossing half-edges in $H(\Phi)$, each crossing two other elements of $H(\Phi)$, then they cannot end at the same edge of $\Phi$.

By definition, conditions (i)-(iii) are satisfied for 'real' faces and half-edges associated with the triple $\left(G, G^{\prime}, \mathcal{D}\right)$, while (iv) follows from Lemma 2.1.

Assume without loss of generality that the boundary of $\Phi$ is a simple cycle. If this is not the case, replace each vertex of $\Phi$ encountered more than once during a full counterclockwise tour around the boundary of $\Phi$ by as many copies as many times it is visited, and replace each edge of $\Phi$ whose sides both belong to $\Phi$ by two edges running very close to it. Obviously, the number of sides of the resulting "face" will be the same as that of the original.

We proceed by induction on $s=|\Phi|$. We start with the case $s=3$. Denote the vertices of $\Phi$ by $A, B$, and $C$. Let $a, b$, and $c$ denote the number of half-edges in $\Phi$, emanating from $A, B$, and $C$, respectively. Without loss of generality, we can assume that $a \geq b \geq c$. By (i), every half-edge must end in the interior of the edge opposite its starting point. Thus, by (ii), we have $a \leq 3$. Every half-edge emanating from $C$ must cross all half-edges emanating from $A$ and $B$. Hence, by (iii), if $a+b>2$, we must have $c=0$. Similarly, if $a=3$, then $b=0$ must hold. The only set of values satisfying the above constraints, for which we have $a+b+c>3 s-6=3$, is $a=b=2$ and $c=0$. In this case both half-edges emanating from $A$ end in the interior of the edge $B C$ and both cross the two half-edges emanating from $B$, which contradicts condition (iv).

Now let $s>3$, and suppose that the statement has already been proved for faces with fewer than $s$ sides.

Given a half-edge $h \in H(\Phi)$, its endpoints divide the boundary of $\Phi$ into two pieces. Consider all of these pieces over all elements of $H(\Phi)$, and let $\mathcal{R}$ be the set of those pieces that have the smallest number of vertices in their interiors. Pick $R$, a minimal 

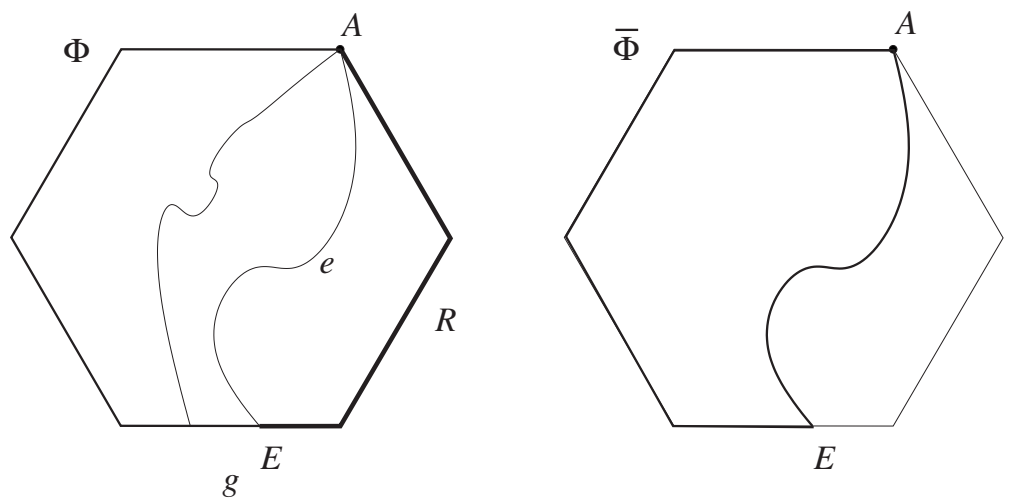

Fig. 3. Induction step in the proof of Lemma 2.2.

element of $\mathcal{R}$ by containment. $R$ is defined by a half-edge $e=A E$, where $A$ is a vertex of $\Phi$ and $E$ is an interior point of an edge $g$ of $\Phi$ (see Fig. 3). Let $P$ denote the set of all half-edges in $\Phi$ that start at $A$ and end on $g$. Clearly, we have $e \in P$ and, by (ii), $1 \leq|P| \leq 3$. By the minimality of $R$, every element of $P$ other than $e$ ends outside $R$. Let $Q$ denote the set of half-edges in $\Phi$ that cross $e$. We claim that every element $h \in Q$ crosses all half-edges in $P$. Indeed, otherwise $h$ would start at an interior vertex of $R$ and end at a point of $g$ outside $R$. However, in this case the piece of the boundary of $\Phi$ defined by $h$, which contains $E$, would have fewer interior vertices than $R$, contradicting the choice of $R$.

Thus, if $|P|=3$ then, by (iii), $Q$ must be empty. If $|P|=2$ then, by (iv), $|Q| \leq 1$, and if $|P|=1$ then, by (iii), $|Q| \leq 2$. Therefore, we always have $|P \cup Q| \leq 3$.

Let $\bar{\Phi}$ denote the "face" obtained from $\Phi$ as follows. Replace the arc $R$ by the halfedge $e$. Remove all vertices and edges in $R$, and regard the union of $e$ and the part of $g$ not belonging to $R$ as a single new edge (see Fig. 3). By the definition of $\mathcal{R}$, the resulting face has $s^{\prime} \geq 3$ sides. By (i), we have $s^{\prime}<s$. Consider the set of half-edges $H(\bar{\Phi})=H(\Phi) \backslash(P \cup Q)$. None of the elements of this set crosses $e$, so, by the minimality of $R$, all of them lie in $\bar{\Phi}$. They meet conditions (i)-(iv), so one can apply the induction hypothesis to conclude that

$$
|H(\Phi)| \leq|H(\bar{\Phi})|+3 \leq\left(3 s^{\prime}-6\right)+3 \leq 3 s-6
$$

as claimed.

Return to the proof of Theorem 1. A simple face $\Phi$ of $G^{\prime}$ is said to be triangular if $|\Phi|=3$, otherwise it is a big face.

By Lemma 2.2 we have $|H(\Phi)| \leq 3$, for any triangular face $\Phi$. A triangular face $\Phi$ is called an $i$-triangle if $|H(\Phi)|=i(0 \leq i \leq 3)$. A 3-triangle is a $3 X$-triangle if one half-edge emanates from each of its vertices. Otherwise, it is a $3 Y$-triangle.

If $\Phi$ is a $3 Y$-triangle, then at least two of its half-edges must end at the same side. The face adjacent to $\Phi$ along this side is called the neighbor of $\Phi$. 
(a)

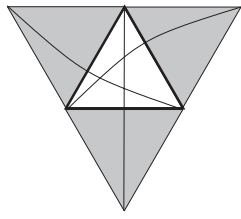

(e)

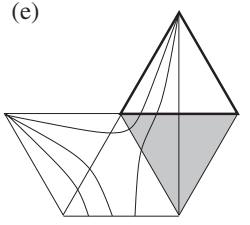

(i)

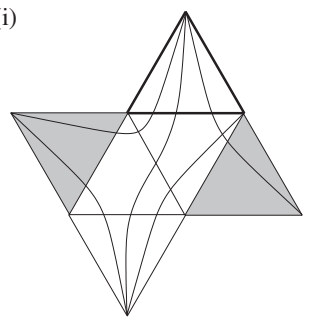

(b)

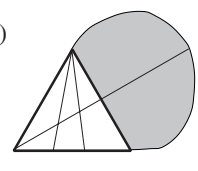

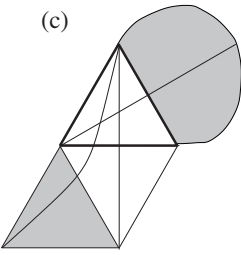

(g)

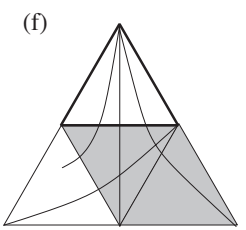

(j)

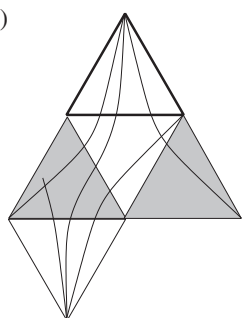

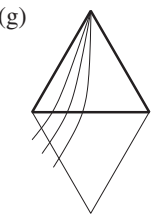

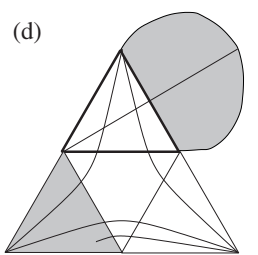

(h)

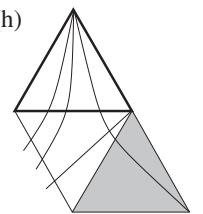

Fig. 4. Proof of Lemma 2.3; triangles that are shaded are not 3-triangles.

An edge of $G-G^{\prime}$ is said to be perfect if it starts and ends in 3-triangles and all the faces it passes through are triangular. The neighbor $\Psi$ of a $3 Y$-triangle $\Phi$ is called a strong neighbor if either it is a 0-triangle or it is a 1-triangle and the extension of one of the half-edges in $H(\Phi)$ ends in $\Psi$.

Lemma 2.3. Let $\Phi$ be a 3-triangle. If the extensions of at least two half-edges in $H(\Phi)$ are perfect, then $\Phi$ is a $3 Y$-triangle with a strong neighbor.

Proof. If $\Phi$ is a $3 X$-triangle, then the extension of none of its half-edges is perfect (see Fig. 4(a)). Indeed, observe that if $\Phi$ is a $3 X$-triangle, then it has three mutually crossing half-edges, so that their extensions do not have any additional crossing and they must end in a face adjacent to $\Phi$. Moreover, no other edges of $G$ can enter a $3 X$-triangle.

Therefore, $\Phi$ is a $3 Y$-triangle. It has a unique neighbor $\Psi$, which, by the assumptions in the lemma, must be a triangle. We use a tedious case analysis, illustrated by Fig. 4, to prove that $\Psi$ is a strong neighbor. We only sketch the argument. The set of extensions of the half-edges in $H(\Phi)$ is denoted by $H$.

Case 1: One half-edge $f \in H(\Phi)$ emanates from a different vertex than the other two. Then the extension $\bar{f} \in H$ of $f$ is not perfect (see Fig. 4(b)). We have to distinguish further cases, depending on where the other two edges end, to conclude that at least one of them cannot be perfect either (see Fig. 4(c),(d)). An interested reader can find a thorough outline of this case in Appendix 1. 


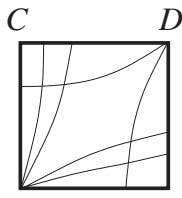

A

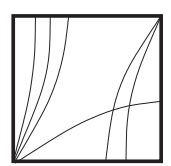

(b)

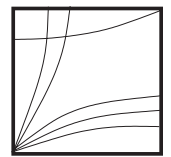

(e)

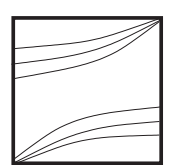

(c)

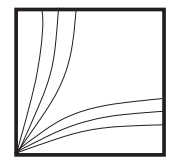

(f)

Fig. 5. Seven different types of quadrilateral faces.

Case 2: All half-edges of $H(\Phi)$ emanate from the same vertex.

Subcase 2.1: Some edge $e \in H$ ends in $\Psi$. Then $\Psi$ is not a 3-triangle, so $e$ is not perfect. If the other two edges are perfect, then $\Psi$ is a 1-triangle (see Fig. 4(e),(f)).

Subcase 2.2: None of the edges in $H$ end in $\Psi$. Suppose $\Psi$ is not a 0 -triangle. Then some edge $e \in H$ must leave $\Psi$ through a different side than the other two edges $f, g \in H$ do (see Fig. 4(g)). Then $e$ cannot be perfect (see Fig. 4(h)). We have to distinguish three cases, depending on whether $f, g$, or neither of them end in the triangle next to $\Psi$. In each of these cases, one can show that $f$ and $g$ cannot be perfect simultaneously (see Fig. 4(i)-(k)).

Claim A. Suppose that $\Psi$ is a simple face of $G^{\prime}$ with $|\Psi|=4$ and $|H(\Psi)|=6$. Then there are seven combinatorially different possibilities for the arrangement of $\Psi$ and the half-edges, as shown in Fig. 5.

The proof of Claim A is a straightforward case analysis, carried out in Appendix 2.

Lemma 2.4. Let $\Psi$ be a simple face of $G^{\prime}$ with $|\Psi|=4$ and $|H(\Psi)|=6$, and suppose that the arrangement of half-edges in $\Psi$ is not homeomorphic with configuration $(\mathrm{g})$ in Fig. 5. Then we have

$$
E(G)<5.5(v(G)-2)
$$

Proof. Notice that one of the diagonals of $\Phi$, denoted by $e=A B$, can be added in the interior of $\Phi$ without creating any crossing with the half-edges in $\Psi$ or with other potentially existing edges of $G-G^{\prime}$ that may enter $\Phi$. Thus, by the maximality of $G$ (more precisely, by the fact that $\left.\left(G, G^{\prime}, \mathcal{D}\right) \in \mathcal{G}^{\prime}\right)$, we may assume that that $A$ and $B$ are connected by an edge $e^{\prime}$ of $G$. Obviously, $e^{\prime}$ must lie entirely outside of $\Psi$. (See Fig. 6 for an illustration.) We may also assume that $e^{\prime} \in G^{\prime}$ and that it does not cross any edge of $G$, otherwise replacing $e^{\prime}$ by $e$ in $G$, we would obtain a contradiction with 

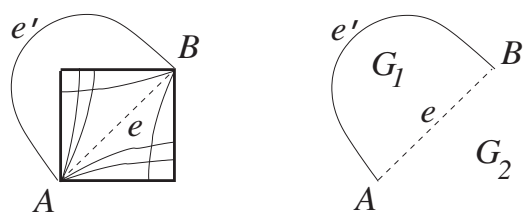

Fig. 6. Proof of Lemma 2.4.

the maximality of $G^{\prime}$ (more precisely, with the fact that $\left(G, G^{\prime}, \mathcal{D}\right) \in \mathcal{G}^{\prime \prime}$ and the total number of crossings along all edges of $G^{\prime}$ is as small as possible).

Let $G_{1}$ (resp. $G_{2}$ ) denote the subgraph of $G$ induced by $A, B$, and all vertices in the interior (resp. exterior) of the "lens" enclosed by $e$ and $e^{\prime}$ (see Fig. 6). Clearly, we have $v(G)=v\left(G_{1}\right)+v\left(G_{2}\right)-2$ and $e(G)=e\left(G_{1}\right)+e\left(G_{2}\right)-1$. As $e^{\prime}$ and $e$ run in the exterior and in the interior of $\Psi$, resp., both $v\left(G_{1}\right)$ and $v\left(G_{2}\right)$ are strictly smaller than $v(G)$. Therefore, we can apply the induction hypothesis to $G_{1}$ and $G_{2}$ to obtain that

$e(G)=e\left(G_{1}\right)+e\left(G_{2}\right)-1 \leq 5.5\left(v\left(G_{1}\right)-2\right)+5.5\left(v\left(G_{2}\right)-2\right)-1<5.5(v(G)-2)$,

as required.

In view of the last lemma, from now on we may and will assume that in every simple quadrilateral face that contains six half-edges, these half-edges form an arrangement homeomorphic to configuration (g) in Fig. 5.

We define a bipartite multigraph $M=\left(V_{1} \cup V_{2}, E\right)$ with vertex classes $V_{1}$ and $V_{2}$, where $V_{1}$ is the set of 3-triangles and $V_{2}$ is the set of all other faces of $G^{\prime}$. For each vertex (3-triangle) $\Phi \in V_{1}$, separately, we add to the edge set $E$ of $M$ some edges incident to $\Phi$, according to the following rules.

- Rule 0: Connect $\Phi$ to an adjacent triangular face $\Psi$ by two parallel edges if $\Psi$ is a 0 -triangle.

- Rule 1: Connect $\Phi$ to any (not necessarily adjacent) 1-triangle $\Psi$ by two parallel edges if there is an edge of $G-G^{\prime}$ that starts in $\Phi$ and ends in $\Psi$.

- Rule 2: Connect $\Phi$ to any (not necessarily adjacent) 2-triangle $\Psi$ by a single edge if there is an edge of $G-G^{\prime}$ that starts in $\Phi$ and ends in $\Psi$.

- Rule 3: If the extension $e$ of a half-edge in $H(\Phi)$ passes through or ends in a big face, we may connect $\Phi$ by a single edge to the first such big face along $e$. However, we use this last rule only to bring the degree of $\Phi$ in $M$ up to 2 . In particular, if we have applied Rules 0 or 1 , for some $\Phi$, we do not apply Rule 3 . Similarly, in no case do we apply Rule 3 for all three half-edges in $H(\Phi)$.

Notice that, besides Rules 0 and 1, the application of Rule 3 can also yield parallel edges if two half-edges in $H(\Phi)$ reach the same big face. However, we never create three parallel edges in $M$.

Let $d(\Phi)$ denote the degree of vertex $\Phi$ in $M$.

Lemma 2.5. For any $\Phi \in V_{1}$, we have $d(\Phi) \geq 2$. 
Proof. We can disregard the restriction on the use of Rule 3, since it only applies if $d(\Phi)$ has already reached 2. If the extension $e$ of a half-edge in $H(\Phi)$ is not perfect, then $e$ yields a (possible) edge of $M$ incident to $\Phi$ according to one of Rules $1-3$. We get two edges this way, unless the extensions of at least two of the half-edges in $H(\Phi)$ are perfect. In this latter case, Lemma 2.3 applies and either Rule 0 or Rule 1 provides two parallel edges of $M$ connecting $\Phi$ to its strong neighbor.

To complete the proof of Theorem 1, we have to estimate from above the degrees of the vertices belonging to $V_{2}$ in $M$. If $\Psi \in V_{2}$ is a 1-triangle or a 2-triangle, we have $d(\Psi) \leq 2$. Every 0 -triangle $\Psi$ is adjacent to at most three 3-triangles, so its degree satisfies $d(\Psi) \leq 6$. The following lemma establishes a bound for big faces.

Lemma 2.6. For any big face $\Psi \in V_{2}$, we have $d(\Psi) \leq 2|\Psi|$. Moreover, if $\Psi$ is a simple quadrilateral face with six half-edges forming an arrangement homeomorphic to the one depicted in Fig. 5(g), we have d $(\Psi) \leq 4$.

Proof. Every edge of $M$ incident to $\Psi$ corresponds to an edge of $G-G^{\prime}$ that starts in some 3-triangle and enters $\Psi$. Different edges of $M$ correspond to different edges of $G-G^{\prime}$ (or opposite orientations of the same edge). Since any side of $\Psi$ crosses at most three edges of $G-G^{\prime}$, we obtain the weaker bound $d(\Psi) \leq 3|\Psi|$. If $\Psi$ is a simple quadrilateral face satisfying the conditions in the second part of the lemma, then two of its sides do not cross any edge of $G-G^{\prime}$, hence we have $d(\Psi) \leq 6$. The stronger bounds stated in the lemma immediately follow from the fact that, even if some side of a big face $\Psi$ is crossed by three edges of $G-G^{\prime}$, they can contribute only at most 2 to the degree of $\Psi$.

To verify this fact, consider a fixed side $g$ of $\Psi$, and suppose that it crosses three edges of $G-G^{\prime}$. These crossings do not contribute to the degree of $\Psi$ if both sides of $g$ belong to the interior of $\Psi$; so we assume that this is not the case. Every edge $e$ that crosses $g$ is divided by $g$ into two pieces. If the piece incident to the exterior side of $g$ passes through a big face or does not end in a 3-triangle, then $e$ does not contribute to $d(\Psi)$. Therefore, we may assume that all three such edge pieces pass through only triangular faces and end in 3-triangles (hence, excluding all but cases (a), (g), (j), and (k) in Fig. 7). A case analysis shows that either at least one of these edge pieces ends in a 3-triangle which has a strong neighbor (see Fig. 7(g),(j),(k)), or all of them end in the same 3-triangle (see Fig. 7(a)). In either case, the corresponding three edges contribute at most two to the degree of $\Psi$.

The details of the case analysis are omitted, but they can be reconstructed from Fig. 7, where the circular arc, together with the horizontal segment, represents the boundary of $\Psi$. Dark-shaded triangles are not 3-triangles, while light-shaded triangles are $3 \mathrm{Y}$ triangles with a strong neighbor. We omitted the cases where the three edges crossing $g$ leave the triangular face adjacent to $g$ through the same other edge $g^{\prime}$. These cases can be handled by removing the edge $g$ and considering the resulting big face and the three edges crossing the side $g^{\prime}$ of this face. Applying this reduction twice if necessary we reduce this case to one of the other cases. 
(a)

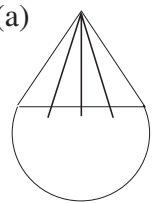

(e)

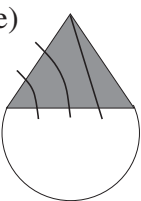

(i)

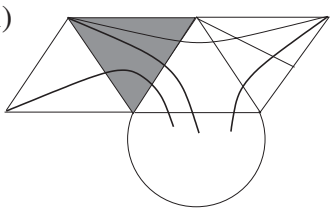

(b)

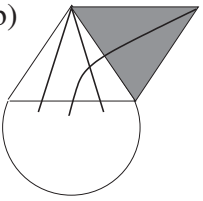

(f)

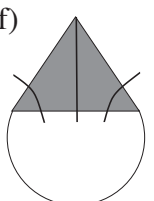

(c)

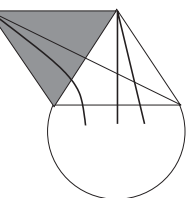

(g)

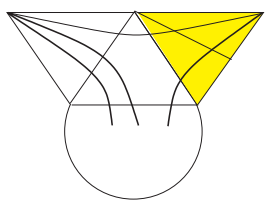

(j)

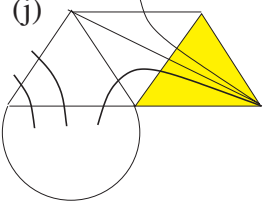

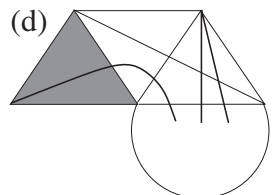

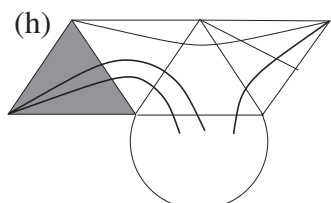

(k)

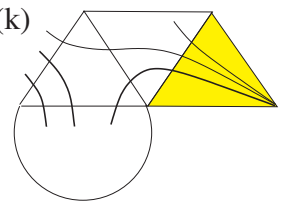

Fig. 7. Proof of Lemma 2.6; dark-shaded triangles (b)-(f), (h), (i) and light-shaded triangles (g), (j), (k).

For any face $\Phi$, let $t(\Phi)$ and $\bar{t}(\Phi)$ denote the number of triangles and diagonals, resp., in a triangulation of $\Phi$. Thus, if the sum of the number of isolated vertices of $G^{\prime}$ that lie in the interior of $\Phi$ and the number of connected components of the boundary of $\Phi$ is $k$, we have $t(\Phi)=|\Phi|+2 k-4$ and $\bar{t}(\Phi)=|\Phi|+3 k-6$.

We introduce the notation $\bar{d}(\Phi):=-d(\Phi)$ for $\Phi \in V_{1}$, and $\bar{d}(\Psi):=d(\Psi)$ for $\Psi \in V_{2}$. Let $V:=V_{1} \cup V_{2}$ denote the set of all faces of $G^{\prime}$. Then the fact that the sum of degrees of the vertices must be the same on both sides of $M$, can be expressed by the equation

$$
\sum_{\Phi \in V} \bar{d}(\Phi)=0
$$

Lemma 2.7. For every face $\Phi \in V$, we have

$$
|H(\Phi)|+\frac{1}{4} \bar{d}(\Phi) \leq \frac{5}{2} t(\Phi)+2 \bar{t}(\Phi) .
$$

Proof. The proof is by straightforward case analysis, based on the previous lemmas.

If $\Phi$ is triangular, we have $\bar{t}(\Phi)=0, t(\Phi)=1$, so that $\frac{5}{2} t(\Phi)+2 \bar{t}(\Phi)=\frac{5}{2}$. For a 3-triangle $\Phi$, by Lemma 2.5 , we have $|H(\Phi)|+\frac{1}{4} \bar{d}(\Phi) \leq 3+\frac{1}{4}(-2)=\frac{5}{2}$. For a 2-triangle $\Phi$, we have $|H(\Phi)|+\frac{1}{4} \bar{d}(\Phi) \leq 2+\frac{1}{4}(2)=\frac{5}{2}$. For a 1-triangle $\Phi$, we have $|H(\Phi)|+\frac{1}{4} \bar{d}(\Phi) \leq 1+\frac{1}{4}(2)=\frac{3}{2}$, and for a 0 -triangle $\Phi$, we have $|H(\Phi)|+\frac{1}{4} \bar{d}(\Phi) \leq$ $0+\frac{1}{4}(6)=\frac{3}{2}$.

If $\Phi$ is a simple face with $|\Phi| \geq 5$ sides, we have $t(\Phi)=|\Phi|-2$ and $\bar{t}(\Phi)=|\Phi|-3$, so that $\frac{5}{2} t(\Phi)+2 \bar{t}(\Phi)=\frac{9}{2}|\Phi|-11$. It follows from Lemmas 2.2 and 2.6 that $|H(\Phi)| \leq$ $3|\Phi|-6$ and $\bar{d}(\Phi)=d(\Phi) \leq 2|\Phi|$. Thus, we have

$$
|H(\Phi)|+\frac{1}{4} \bar{d}(\Phi) \leq \frac{7}{2}|\Phi|-6 \leq \frac{9}{2}|\Phi|-11 .
$$


If $\Phi$ is a simple face with $|\Phi|=4$, we have $t(\Phi)=2, \bar{t}(\Phi)=1$, so that $\frac{5}{2} t(\Phi)+$ $2 \bar{t}(\Phi)=7$. By Lemmas 2.2 and 2.6, we obtain $|H(\Phi)| \leq 6$ and $\bar{d}(\Phi)=d(\Phi) \leq 8$. If $|H(\Phi)| \leq 5$, then $|H(\Phi)|+\frac{1}{4} \bar{d}(\Phi) \leq 5+\frac{1}{4}(8)=7$. If $|H(\Phi)|=6$, then by Lemma $2.6 \bar{d}(\Phi)=d(\Phi) \leq 4$ and $|H(\Phi)|+\frac{1}{4} \bar{d}(\Phi) \leq 6+\frac{1}{4}(4)=7$.

Finally, assume that $\Phi$ is not a simple face, i.e., its boundary is not connected or it contains at least one isolated vertex of $G^{\prime}$ in its interior. In this case we have $t(\Phi) \geq|\Phi|$, $\bar{t}(\Phi) \geq|\Phi|$, so that $\frac{5}{2} t(\Phi)+2 \bar{t}(\Phi) \geq \frac{9}{2}|\Phi|$. By Lemma 2.6, we now obtain $\bar{d}(\Phi)=$ $d(\Phi) \leq 2 \Phi$. Lemma 2.2 does not apply here, but we have $|H(\Phi)| \leq 3|\Phi|$, because every half-edge in $H(\Phi)$ ends at an edge of $\Phi$. Hence, we have $|H(\Phi)|+\frac{1}{4} \bar{d}(\Phi) \leq$ $3|\Phi|+\frac{1}{4}(2|\Phi|)=\frac{7}{2}|\Phi|$.

Now we can easily complete the proof of Theorem 1 . Since every edge of $G-G^{\prime}$ gives rise to two half-edges, we have

$e(G)-e\left(G^{\prime}\right)=\frac{1}{2} \sum_{\Phi \in V}|H(\Phi)|=\frac{1}{2} \sum_{\Phi \in V}\left(|H(\Phi)|+\frac{1}{4} \bar{d}(\Phi)\right) \leq \frac{5}{4} \sum_{\Phi \in V} t(\Phi)+\sum_{\Phi \in V} \bar{t}(\Phi)$,

where the inequality holds by Lemma 2.7 . We obviously have that $\sum_{\Phi \in V} t(\Phi)=$ $2(v(G)-2)$, which is equal to the total number of faces in any triangulation of $G^{\prime}$. In order to obtain such a triangulation from $G^{\prime}$, one needs to add $\sum_{\Phi \in V} \bar{t}(\Phi)$ edges. Hence, we have $\sum_{\Phi \in V} \bar{t}(\Phi)=3(v(G)-2)-e\left(G^{\prime}\right)$. Notice that triangulating each face separately may create a triangulation of the plane containing some parallel edges, but this has no effect on the number of triangles or the number of edges. Now the theorem follows by simple calculation:

$$
\begin{aligned}
e(G) & =e\left(G^{\prime}\right)+\left(e(G)-e\left(G^{\prime}\right)\right) \leq e\left(G^{\prime}\right)+\frac{5}{4} \cdot 2(v(G)-2)+\left(3(v(G)-2)-e\left(G^{\prime}\right)\right) \\
& =5.5(v(G)-2) .
\end{aligned}
$$

This completes the proof of the inequality in Theorem 1.

We close this section by presenting a construction which shows that the result is not far from being tight.

Proposition 2.8. For every $v \equiv 0(\bmod 6), v \geq 12$, there exists a graph $G$ with $v$ vertices and 5.5 $(v-2)-4$ edges that can be drawn in the plane so that each of its edges crosses at most three others. That is, for these values we have $e_{3}(v) \geq 5.5 v-15$.

Proof. Let $T_{q}$ denote a hexagonal tiling of a vertical cylindrical surface with $q \geq 1$ horizontal layers, each consisting of three hexagonal faces wrapped around the cylinder (see Fig. 8). Notice that the top and the bottom face of the cylinder are also hexagonal. Let $V_{q}$ be the set of all the vertices of the tiles. To each face except the top and the bottom one, add eight diagonals (all but one main diagonal). Finally, add all diagonals to the top and the bottom face that do not yield parallel edges. This means adding six edges on both the top and the bottom face, as depicted in Fig. 8. The resulting graph $G_{q}$ is drawn on the surface of the cylinder with each edge crossing at most three other edges. We have $v\left(G_{q}\right)=6 q+6$ and $e\left(G_{q}\right)=33 q+18=5.5 v\left(G_{q}\right)-15$. 

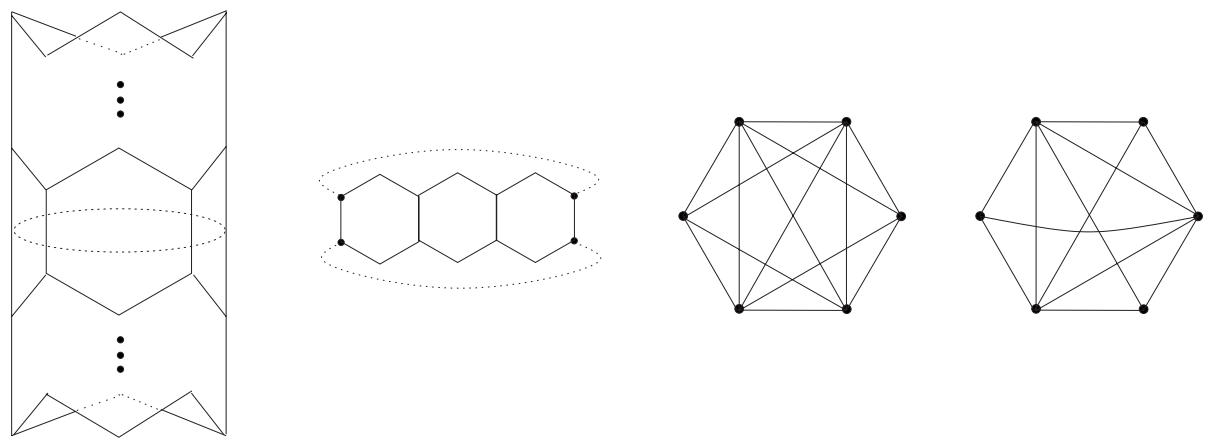

Fig. 8. The vertical cylindrical surface, its layer, side-face, and top/bottom face.

\section{Proof of Theorem 2}

For any graph $G$ drawn in the plane, let $G^{\text {free }}$ denote the subgraph of $G$ on the same vertex set, consisting of all crossing-free edges. Let $\triangle\left(G^{\text {free }}\right)$ denote the number of triangular faces of $G^{\text {free }}$, containing no vertex of $G$ in their interiors.

Lemma 3.1. Let $G$ be a graph on $v(G) \geq 3$ vertices, which is drawn in the plane so that none of its edges crosses two others. Then the number of edges of $G$ satisfies is

$$
e(G) \leq 4(v(G)-2)-\frac{1}{2} \triangle\left(G^{\text {free }}\right) .
$$

Proof. We can assume without loss of generality that $G^{\text {free }}$ is maximal in the following sense: if two vertices, $u$ and $v$, can be connected by a Jordan arc that does not cross any edge of $G$, then $G^{\text {free }}$ contains an edge $u v$ between these vertices. We can also assume that $G$ is 3-connected. Otherwise, we can conclude by induction on $v(G)$, as follows. Let $G=G_{1} \cup G_{2}$ be a decomposition of $G$ into subgraphs on fewer than $v(G)$ vertices, where $G_{1}$ and $G_{2}$ share at most two vertices. Clearly, we have $\left(v\left(G_{1}\right)-2\right)+\left(v\left(G_{2}\right)-2\right) \leq$ $v(G)-2, e\left(G_{1}\right)+e\left(G_{2}\right) \geq e(G)$, and $\Delta\left(G_{1}^{\text {free }}\right)+\Delta\left(G_{2}^{\text {free }}\right) \geq \Delta\left(G^{\text {free }}\right)$. Therefore, applying the induction hypothesis to $G_{1}$ and $G_{2}$ separately, we obtain that the statement of the lemma holds for $G$.

Observe that if two edges $u v$ and $z w$ cross each other, then $u$ and $z$, say, can be connected by a Jordan arc running very close to the union of the edges $u v$ and $z v$, without crossing any edge of $G$. Thus, it follows from the maximality of $G^{\text {free }}$ that $u z$, and similarly $z v, v w$, and $w u$, are edges of $G^{\text {free }}$. Moreover, the quadrilateral $u z v w$ containing the crossing pair of edges $u v, z w$ must be a face of $G^{\text {free }}$. To see this, it is enough to observe that the 3-connectivity of $G$ implies that this quadrilateral cannot contain any vertex of $G$ in its interior. Thus, all edges in $G-G^{\text {free }}$ are diagonals of quadrilateral faces of $G^{\text {free }}$. Letting $q\left(G^{\text {free }}\right)$ denote the number of quadrilateral faces of $G^{\text {free }}$, we obtain

$$
e\left(G^{\text {free }}\right)+2 q\left(G^{\text {free }}\right)-e(G) \geq 0 .
$$

Let $f\left(G^{\text {free }}\right)$ denote the total number of faces of $G^{\text {free }}$. Then we have

$$
f\left(G^{\text {free }}\right)-q\left(G^{\text {free }}\right)-\triangle\left(G^{\text {free }}\right) \geq 0
$$


and, by Euler's formula,

$$
v(G)+f\left(G^{\text {free }}\right)-e\left(G^{\text {free }}\right)-2 \geq 0 .
$$

Double counting the pairs $(\sigma, a)$, where $\sigma$ is a face of $G^{\text {free }}$ and $a$ is an edge of $\sigma$, we obtain

$$
2 e\left(G^{\text {free }}\right)-4 f\left(G^{\text {free }}\right)+\triangle\left(G^{\text {free }}\right) \geq 0 .
$$

Multiplying the above four inequalities by the coefficients $1,2,4$, and $\frac{3}{2}$, respectively, and adding them, the lemma follows.

Instead of Theorem 2, we establish a slightly stronger claim.

Lemma 3.2. Let $G$ be a graph on $v(G) \geq 3$ vertices, which is drawn in the plane with $x(G)$ crossings. Then we have

$$
x(G) \geq \frac{7}{3} e(G)-\frac{25}{3}(v(G)-2)+\frac{2}{3} \triangle\left(G^{\text {free }}\right) .
$$

Proof. We use induction on $x(G)+v(G)$. As in the proof of Lemma 3.1, we can assume that $G$ is 3-connected and that $G^{\text {free }}$ is maximal in the sense that whenever the points $u$ and $v$ can be connected by a Jordan arc without crossing any edge of $G$, the edge $u v$ belongs to $G^{\text {free }}$. We distinguish four cases.

Case 1: G contains an edge that crosses at least three other edges. Let $a$ be such an edge, and let $G_{0}$ be the subgraph of $G$ obtained by removing $a$. Now we have $e\left(G_{0}\right)=e(G)-1$, $x\left(G_{0}\right) \leq x(G)-3$, and $\triangle\left(G_{0}^{\text {free }}\right) \geq \triangle\left(G^{\text {free }}\right)$. Applying the induction hypothesis to $G_{0}$, we get

$$
x(G)-3 \geq \frac{7}{3}(e(G)-1)-\frac{25}{3}(v(G)-2)+\frac{2}{3} \triangle\left(G^{\text {free }}\right),
$$

which implies the statement of the lemma.

Case 2: Every edge in $G$ crosses at most one other edge. Lemma 3.1 yields

$$
4(v(G)-2)-\frac{1}{2} \triangle\left(G^{\text {free }}\right) \geq e(G) .
$$

The statement is obtained by multiplying this inequality by $\frac{4}{3}$ and adding to it the simple inequality $x(G) \geq e(G)-3(v(G)-2)$ mentioned in the Introduction.

Case 3: There exists an edge e of $G$ that crosses two other edges, one of which does not cross any other edge of $G$. Let $z w$ be an edge that crosses $e$ at point $x$ and does not participate in any other crossing. Let $u$ denote the endpoint of $e$ for which the piece of $e$ between $x$ and $u$ is crossing-free (Fig. 9). Notice that $u$ can be connected to both $z$ and $w$ by non-crossing Jordan arcs, without crossing any edge of $G$. Therefore, by the maximality of $G^{\text {free }}$, the edges $u z$ and $u w$ must belong to $G^{\text {free }}$. Let $G_{0}$ be the subgraph of $G$ obtained by removing the edge $e$. We have $e\left(G_{0}\right)=e(G)-1$ and $x\left(G_{0}\right)=x(G)-2$. Clearly, $G_{0}^{\text {free }}$ contains $z w$ and all edges in $G^{\text {free }}$. By the 3-connectivity of $G$, the triangle $u z w$ must be a triangular face of $G_{0}^{\text {free }}$, so that we have $\Delta\left(G_{0}^{\text {free }}\right) \geq \Delta\left(G^{\text {free }}\right)+1$. Applying the induction hypothesis to $G_{0}$, we obtain

$$
x(G) \geq \frac{7}{3} e(G)-\frac{25}{3}(v(G)-2)+\frac{2}{3} \triangle\left(G^{\text {free }}\right)+\frac{1}{3},
$$

which is better than what we need. 


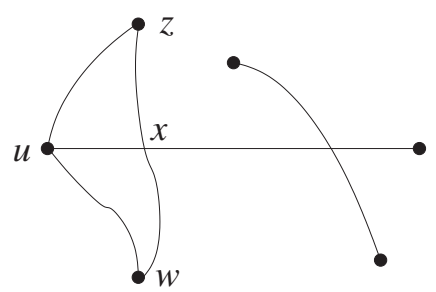

Fig. 9. Proof of Lemma 3.2, Case 3.

Case 4: There exists an edge a of $G$ that crosses precisely two other edges, $b$ and $c$, and each of these edges also participates in precisely two crossings.

Subcase 4.1: $b$ and $c$ do not cross each other. Let $G_{0}$ be the subgraph of $G$ obtained by removing $b$. Clearly, we have $e\left(G_{0}\right)=e(G)-1, x\left(G_{0}\right)=x(G)-2$, and $\triangle\left(G_{0}^{\text {free }}\right) \geq$ $\triangle\left(G^{\text {free }}\right)$. Notice that $c$ is an edge of $G_{0}$ that crosses two other edges; one of them is $a$, which is crossed by no other edge of $G_{0}$ (Fig. 10). Thus, we can apply to $G_{0}$ the last inequality in the analysis of Case 3 to conclude that

$$
x(G)-2 \geq \frac{7}{3}(e(G)-1)-\frac{25}{3}(v(G)-2)+\frac{2}{3} \triangle\left(G^{\mathrm{free}}\right)+\frac{1}{3},
$$

which is precisely what we need.

Subcase 4.2: $b$ and c cross each other. The three crossing edges, $a, b$, and $c$, can be drawn on the sphere in two topologically different ways. If the closed curve formed by segments of the three edges separates two of the endpoints of the three edges from the other four, then the graph is not 3-connected as the vertices on the two sides of this closed curve are only connected by two edges (see the configuration on the left-hand side of Fig. 11). So it is enough to consider the configuration depicted on the right-hand side of Fig. 11. By the maximality condition, $G^{\text {free }}$ must contain the six dashed edges in the figure. Note that $a, b$, and $c$ are not crossed by any additional edges, so all other edges of $G$ contained in the hexagon $\Phi$, formed by the dashed edges, must be contained in one of the triangular or quadrilateral faces of the arrangement, and the existence of such edges contradicts the 3-connectedness of $G$. Thus, $\Phi$ is a face of $G^{\text {free }}$, and the only edges of $G$ inside this face are $a, b$, and $c$. Let $G_{0}$ be the graph obtained from $G$ by removing the edges $a, b, c$, and inserting a new vertex in the interior of $\Phi$, which is

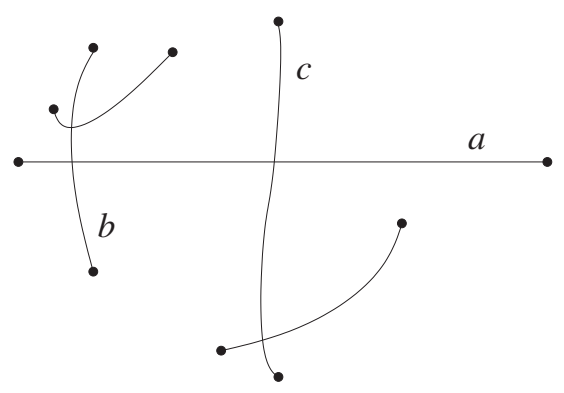

Fig. 10. Proof of Lemma 3.2, Subcase 4.1. 

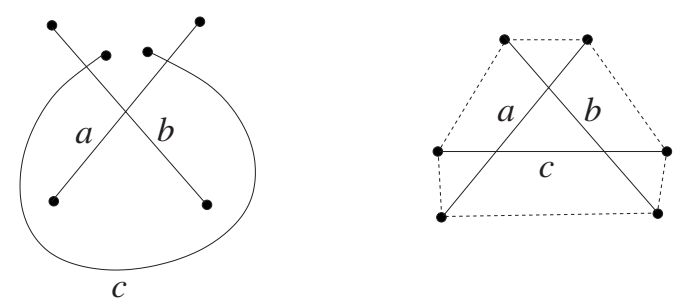

Fig. 11. Proof of Lemma 3.2, Subcase 4.2.

connected to every vertex of $\Phi$ by crossing-free edges. We have $v\left(G_{0}\right)=v(G)+1$ and $x\left(G_{0}\right)=x(G)-3$, so that we can apply the induction hypothesis to $G_{0}$. Obviously, we have $e\left(G_{0}\right)=e(G)+3$ and $\triangle\left(G_{0}^{\text {free }}\right)=\Delta\left(G^{\text {free }}\right)+6$. Thus, we obtain

$$
x(G)-3 \geq \frac{7}{3}(e(G)+3)-\frac{25}{3}(v(G)-1)+\frac{2}{3}\left(\triangle\left(G^{\text {free }}\right)+6\right),
$$

which is much stronger than the inequality in the lemma.

The tightness of Theorem 2 is discussed at the end of the last section.

\section{Proof of Theorem 3}

Our proof is based on the following consequence of Theorems 1 and 2.

Corollary 4.1. The crossing number of any graph $G$ of at least three vertices satisfies

$$
\operatorname{cr}(G) \geq 4 e(G)-\frac{103}{6}(v(G)-2) .
$$

Proof. If $G$ has at most $5(v(G)-2)$ edges, then the statement directly follows from Theorem 2. If $G$ has more than $5(v(G)-2)$ edges, fix one of its drawings in which the number of crossings is minimum. Delete the edges of $G$ one by one until we obtain a graph $G_{0}$ with $5(v(G)-2)$ edges. At each stage, delete one of the edges that participates in the largest number of crossings in the current drawing. Using the inequality $e_{2}(v) \leq 5(v-2)$ proved in [PTo1] and quoted in Section 1, at the time of its removal every edge has at least three crossings. Moreover, by Theorem 1, with the possible exception of the at most $\frac{1}{2}(v(G)-2)$ edges deleted last, every edge has at least four crossings. Thus, the total number of deleted crossings is at least

$$
4(e(G)-5(v(G)-2))-\frac{1}{2}(v(G)-2)=4 e(G)-\frac{41}{2}(v(G)-2) .
$$

On the other hand, applying Theorem 2 to $G_{0}$, we obtain that the number of crossings not removed during the algorithm is at least

$$
\operatorname{cr}\left(G_{0}\right) \geq \frac{10}{3}(v(G)-2) .
$$

Summing up these two estimates, the result follows. 
Now we can easily complete the proof of Theorem 3 . Let $G$ be a graph drawn in the plane with $\operatorname{cr}(G)$ crossings, and suppose that $e(G) \geq \frac{103}{16} v(G)$.

Construct a random subgraph $G^{\prime} \subseteq G$ by selecting each vertex of $G$ independently with probability

$$
p=\frac{103}{16} \frac{v(G)}{e(G)} \leq 1,
$$

and letting $G^{\prime}$ be the subgraph of $G$ induced by the selected vertices. The expected number of vertices of $G^{\prime}$ is $E\left[v\left(G^{\prime}\right)\right]=p v(G)$. Similarly, $E\left[e\left(G^{\prime}\right)\right]=p^{2} e(G)$. The expected number of crossings in the drawing of $G^{\prime}$ inherited from $G$ is $p^{4} \operatorname{cr}(G)$, and the expected value of the crossing number of $G^{\prime}$ is even smaller.

By Corollary $4.1, \operatorname{cr}\left(G^{\prime}\right) \geq 4 e\left(G^{\prime}\right)-\frac{103}{6} v\left(G^{\prime}\right)$ holds for every $G^{\prime}$. (Note that after eliminating the constant term in Corollary 4.1 , we do not have to assume any more that $v\left(G^{\prime}\right) \geq 3$; the above inequality is true for every $G^{\prime}$.) Taking expectations, we obtain

$$
p^{4} \operatorname{cr}(G) \geq E\left[\operatorname{cr}\left(G^{\prime}\right)\right] \geq 4 E\left[e\left(G^{\prime}\right)\right]-\frac{103}{6} E\left[v\left(G^{\prime}\right)\right]=4 p^{2} e(G)-\frac{103}{6} p v(G) .
$$

This implies that

$$
\operatorname{cr}(G) \geq \frac{1024}{31827} \frac{e^{3}(G)}{v^{2}(G)} \geq \frac{1}{31.1} \frac{e^{3}(G)}{v^{2}(G)},
$$

provided that $e(G) \geq \frac{103}{16} v(G)$.

To obtain an unconditional lower bound on the crossing number of any graph $G$, we need different estimates when $e(G)<\frac{103}{16} v(G)$. Comparing the bounds in Theorem 2 and in Corollary 4.1 with the trivial estimates $\operatorname{cr}(G) \geq 0$ and $\operatorname{cr}(G) \geq e-3(v(G)-2)$, a case analysis shows that

$$
\frac{1024}{31827} \frac{e^{3}(G)}{v^{2}(G)}-\operatorname{cr}(G) \leq 1.06 v(G) .
$$

The maximum is attained for a graph $G$ with $e(G)=4(v(G)-2)$ and $\operatorname{cr}(G)=v(G)-2$. In conclusion,

$$
\operatorname{cr}(G) \geq \frac{1024}{31827} \frac{e^{3}(G)}{v^{2}(G)}-1.06 v(G) \geq \frac{1}{31.1} e^{3}(G) v^{2}(G)-1.06 v(G)
$$

holds for every graph $G$. This completes the proof of Theorem 3 .

Remark 4.2. Pach and Tóth [PTo2] introduced two variants of the crossing number. The pairwise crossing number (resp. the odd crossing number) of $G$ is defined as the minimum number of pairs of non-adjacent edges that cross (resp. cross an odd number of times) over all drawings of $G$. These parameters are at most as large as $\operatorname{cr}(G)$. It is known that the odd crossing number is smaller than the other two for certain graphs [PSS], but one cannot rule out the possibility that the pairwise crossing number is always equal to $\operatorname{cr}(G)$. The original proofs of the Crossing Lemma readily generalize to the new crossing numbers, and it follows that both of them are at least $\frac{1}{64}\left(e^{3}(G) / v^{2}(G)\right)$, provided that $e(G) \geq 4 v(G)$. We have been unable to extend our proof of Theorem 3 to these parameters. 


\section{Applications, Problems, Remarks}

Every improvement of the Crossing Lemma automatically leads to improved bounds in all of its applications. For completeness and future reference, we include some immediate corollaries of Theorem 3 with a sketch of computations.

First, we plug Theorem 3 into Székely's method [Sz] to improve the coefficient of the main term in the Szemerédi-Trotter theorem $[\mathrm{SzT}],\left[\mathrm{CE}^{+}\right],[\mathrm{PTo} 1]$.

Corollary 5.1. Given $m$ points and $n$ lines in the Euclidean plane, the number of incidences between them is at most $2.5 m^{2 / 3} n^{2 / 3}+m+n$.

Proof. We can assume that every line and every point is involved in at least one incidence, and that $n \geq m$, by duality. Since the statement is true for $m=1$, we have to check it only for $m \geq 2$.

Define a graph $G$ drawn in the plane such that the vertex set of $G$ is the given set of $m$ points, and join two points with an edge drawn as a straight-line segment if the two points are consecutive along one of the lines. Let $I$ denote the total number of incidences between the given $m$ points and $n$ lines. Then $v(G)=m$ and $e(G)=I-n$. Since every edge belongs to one of the $n$ lines, $\operatorname{cr}(G) \leq\left(\begin{array}{l}n \\ 2\end{array}\right)$. Applying Theorem 2 to $G$, we obtain that $(1 / 31.1)\left((I-n)^{3} / m^{2}\right)-1.06 m \leq \operatorname{cr}(G) \leq\left(\begin{array}{c}n \\ 2\end{array}\right)$. Using that $n \geq m \geq 2$, easy calculation shows that

$$
I-n \leq \sqrt[3]{15.55 m^{2} n^{2}+33 m^{3}} \leq \sqrt[3]{15.55} n^{2 / 3} m^{2 / 3}+m,
$$

which implies the statement.

It was shown in [PTo1] that Corollary 5.1 does not remain true if we replace the constant 2.5 by 0.42 .

Theorem 3 readily generalizes to multigraphs with bounded edge multiplicity, improving the constant in Székely's result [Sz].

Corollary 5.2. Let $G$ be a multigraph with maximum edge multiplicity $m$. Then

$$
\operatorname{cr}(G) \geq \frac{1}{31.1} \frac{e^{3}(G)}{m v^{2}(G)}-1.06 m^{2} v(G)
$$

Proof. Define a random simple subgraph $G^{\prime}$ of $G$ as follows. For each pair of vertices $v_{1}, v_{2}$ of $G$, let $e_{1}, e_{2}, \ldots, e_{k}$ be the edges connecting them. With probability $1-k / m$, $G^{\prime}$ will not contain any edge between $v_{1}$ and $v_{2}$. With probability $\mathrm{k} / \mathrm{m}, G^{\prime}$ contains precisely one such edge, and the probability that this edge is $e_{i}$ is $1 / m(1 \leq i \leq k)$. Applying Theorem 3 to $G^{\prime}$ and taking expectations, the result follows.

Next, we state here the improvement of another result in [PTo1].

Corollary 5.3. Let $G$ be a graph drawn in the plane so that every edge is crossed by at most $k$ others, for some $k \geq 1$, and every pair of edges has at most one point in common. 
Then

$$
e(G) \leq 3.95 \sqrt{k} v(G)
$$

Proof. For $k \leq 2$, the result is weaker than the bounds given in [PTo1]. Assume that $k \geq 3$, and consider a drawing of $G$ such that every edge crosses at most $k$ others. Let $x$ denote the number of crossings in this drawing. If $e(G)<\frac{103}{6} v(G)$, then there is nothing to prove. If $e(G) \geq \frac{103}{6} v(G)$, then using Theorem 3, we obtain

$$
\frac{1024}{31827} \frac{e^{3}(G)}{v^{2}(G)} \leq \operatorname{cr}(G) \leq x \leq \frac{e(G) k}{2},
$$

and the result follows.

Recall that $e_{k}(v)$ was defined as the maximum number of edges that a graph of $v$ vertices can have if it can be drawn in the plane with at most $k$ crossings per edge. We define some other closely related functions. Let $e_{k}^{*}(v)$ denote the maximum number of edges of a graph of $v$ vertices which has a drawing that satisfies the above requirement and, in addition, every pair of its edges meets at most once (either at an endpoint or at a proper crossing). We define $\bar{e}_{k}(v)$ and $\bar{e}_{k}^{*}(v)$ analogously, with the only difference that now the maximums are taken over all triangle-free graphs with $v$ vertices.

It was mentioned in the Introduction (see Lemma 1.1) that $e_{k}(v)=e_{k}^{*}(v)$ for $0 \leq$ $k \leq 3$, and that $e_{k}^{*}(v) \leq(k+3)(v-2)$ for $0 \leq k \leq 4$ [PTo1]. For $0 \leq k \leq 2$, the last inequality is tight for infinitely many values of $v$. Our Theorem 1 shows that this is not the case for $k=3$.

Conjecture 5.4. We have $e_{k}(v)=e_{k}^{*}(v)$ for every $k$ and $v$.

Using the proof technique of Theorem 1, it is not hard to improve the bound $e_{4}^{*}(v) \leq$ $7(v-2)$. In particular, in this case Lemma 2.2 holds with $3(|\Phi|-2)$ replaced by $4(|\Phi|-2)$. Moreover, an easy case analysis shows that every triangular face $\Phi$ with four half-edges satisfies at least one of the following two conditions:

1. The extension of at least one of the half-edges in $\Phi$ either ends in a triangular face with fewer than four half-edges, or enters a big face.

2. $\Phi$ is adjacent to an empty triangle.

Based on this observation, one can modify the arguments in Section 2 to obtain the upper bound $e_{4}^{*}(v) \leq\left(7-\frac{1}{9}\right) v-O(1)$.

Conjecture 5.5. $e_{4}^{*}(v) \leq 6 v-O(1)$.

As for the other two functions, we have $\bar{e}_{k}(v)=\bar{e}_{k}^{*}(v)$ for $0 \leq k \leq 3$, and $\bar{e}_{k}^{*}(v) \leq$ $(k+2)(v-2)$ for $0 \leq k \leq 2$. If $0 \leq k \leq 1$, these bounds are attained for infinitely many values of $v$. These estimates were applied by Czabarka et al. $\left[\mathrm{CS}^{+}\right]$to obtain some lower bounds on the so-called biplanar crossing number of complete graphs.

Given a triangle-free graph drawn in the plane so that every edge crosses at most two others, an easy case analysis shows that each quadrilateral face that contains four 


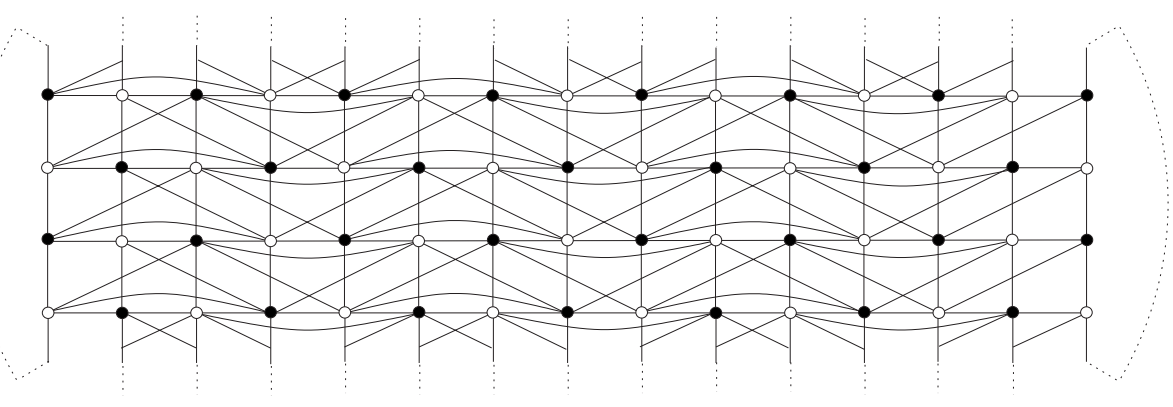

Fig. 12. $\bar{e}_{2}(v) \geq 3.5 v-16$.

half-edges is adjacent to a face which is either non-quadrilateral or does not have four half-edges. ${ }^{1}$ As in the proof of Theorem 1 (before Lemma 2.5), we can use a properly defined bipartite multigraph $M$ to establish the bound

$$
\bar{e}_{2}(v) \leq\left(4-\frac{1}{10}\right) v-O(1) .
$$

Conjecture 5.6. $\quad \bar{e}_{2}(v) \leq 3.5 v-O(1)$.

The coefficient 3.5 in the above conjecture cannot be improved as shown by the triangle-free (actually bipartite!) graph in Fig. 12, whose vertex set is the set of vertices of a $4 \times v / 4$ grid.

Let $\operatorname{cr}(v, e)$ denote the minimal crossing number of a graph with $v \geq 3$ vertices and $e$ edges. Clearly, we have $\operatorname{cr}(v, e)=0$, whenever $e \leq 3(v-2)$, and $\operatorname{cr}(v, e)=e-3(v-2)$ for $3(v-2) \leq e \leq 4(v-2)$. To see that these values are indeed attained by the function, consider the graph constructed in [PTo1], which (if $v$ is a multiple of 4) can be obtained from a planar graph with $v$ vertices, $2(v-2)$ edges, and $v-2$ quadrilateral faces, by adding the diagonals of the faces. If $e<4(v-2)$, delete as many edges participating in a crossing, as necessary.

In the next interval, i.e., when $4(v-2) \leq e \leq 5(v-2)$, Theorem 2 gives a tight bound on $\operatorname{cr}(v, e)$ up to an additive constant. To see this, consider a planar graph with only pentagonal and quadrilateral faces and add all diagonals in every face. If no two faces of the original planar graph share more than a vertex or an edge, for the resulting graph the inequality of Theorem 2 holds with equality. For certain values of $v$ and $e$, no such construction exists, but we only lose a constant.

If $5(v-2) \leq e \leq 5.5(v-2)$, the best known bound, $\operatorname{cr}(v, e) \geq 3 e-\frac{35}{3}(v-2)$, follows from Theorem 2, while for $e \geq 5.5(v-2)$ the best known bound is either the one in Corollary 4.1 or the one in Theorem 3. We do not believe that any of these bounds are optimal.

Conjecture 5.7. $\operatorname{cr}(v, e) \geq \frac{25}{6} e-\frac{35}{2}(v-2)$.

\footnotetext{
${ }^{1}$ This statement actually holds under the assumption that $G$ and $G^{\prime}$ are maximal, in the sense described at the beginning of Section 2 .
} 
Note that, if true, this bound is tight up to an additive constant for $5(v-2) \leq e \leq$ $6(v-2)$. To see this, consider a planar graph with only pentagonal and hexagonal faces and add all diagonals of all faces. If no two faces of the planar graph share more than a vertex or an edge, the resulting graph shows that Conjecture 5.7 cannot be improved. As a first step toward settling this conjecture, we can show the following statement, similar to Lemma 3.1.

Lemma 5.8. Let $G$ be a graph on $v(G) \geq 3$ vertices drawn in the plane so that every edge is involved in at most two crossings. Then

$$
e(G) \leq 5(v(G)-2)-\triangle\left(G^{\text {free }}\right) .
$$

\section{Appendix 1. Case 1 in the Proof of Lemma 2.3}

Our proof will be a straightforward case analysis. Recall that $\Phi$ is a $3 Y$-triangle with the unique neighbor $\Psi$, which is also a triangle. Let $A, B$, and $C$ be the vertices of $\Phi$, and let $f, g$, and $h$ denote the half-edges in $H(\Phi)$. Here, $g$ and $h$ emanate from vertex $A$, while $f$ starts at vertex $B$. Next, we introduce a new notation: given a vertex $V$ in a face $\Upsilon$ of $G^{\prime}$, let $d_{\Upsilon}(V)$ denote the number of half-edges in $H(\Upsilon)$ that emanate from $V$.

Let $\Xi$ denote the face of $G^{\prime}$ that is adjacent to $\Phi$ along side $A C$. First, we claim that the extension $\bar{f} \in H$ of $f$ is not perfect. Indeed, otherwise $\Xi$ is a triangle and edge $\bar{f}$, having crossed half-edges $g$ and $h$, as well as side $A C$, must end in $D$, the vertex of $\Xi$ opposite to $A C$. Since $\bar{f}=B D$ cannot be crossed by any other edge, we have $d_{\Xi}(A)=d_{\Xi}(C)=0$. Aside from $\bar{f}$, the extension of any half-edge in $H(\Xi)$, that emanates from vertex $D$, has to exit $\Xi$ through side $A C$ and enter $\Phi$. It cannot exit $\Phi$ through side $A B$ (it would cross four edges $A C, h, g$, and $A B$, in this order), nor can it end at vertex $B$ (there are no parallel edges). Hence, it must exit $\Phi$ through side $B C$, which is already crossed by the extensions of $g$ and $h$ (see Fig. 13(i)). Therefore, $d_{\Xi}(D) \leq 2$, and $\Xi$ is not a 3-triangle, contradicting the assumption that $\bar{f}$ is perfect.

Next, suppose that the extensions $\bar{g}$ and $\bar{h}$ of $g$ and $h$, respectively, are perfect. We distinguish two cases, based on where these two edges end.

Subcase 1.1: $\bar{h}$ ends in $E$, the vertex of $\Psi$ opposite to BC (see Fig. 13(ii)). Since there are no parallel edges, $\bar{g}$ has to exit $\Phi$ across the side $B E$. Having already crossed three other edges, $\bar{g}$ must end in $\Gamma$, the face of $G^{\prime}$ adjacent to $\Psi$ along $B E$. By the assumption that $\bar{g}$ is perfect, we conclude that $\Gamma$ is a triangle, and we let $F$ be the vertex of $\Gamma$, where $\bar{g}$ ends. Since $\bar{g}$ cannot be crossed by any other edge, then $d_{\Gamma}(B)=d_{\Gamma}(E)=0$.

Aside from $\bar{g}$, the extension of any half-edge in $H(\Gamma)$, that emanates from vertex $F$, exits $\Gamma$ through side $B E$ and enters $\Psi$. It cannot exit $\Psi$ through side $B C$, since $\bar{f}$ already crosses three other edges. Thus, it has to cross $\bar{h}$, that is already crossed by $f$ and $B C$ (see Fig. 13(ii)). Therefore, $d_{\Gamma}(F) \leq 2$, and $\Gamma$ is not a 3-triangle, contradicting the assumption that $\bar{g}$ is perfect.

The symmetric case, when $\bar{g}$ ends in $E$ (and $\bar{h}$ exits $\Psi$ through side $C E$ ), can be handled similarly.

Subcase 1.2: $\bar{g}$ exits $\Psi$ through $B E$ and $\bar{h}$ exits $\Psi$ through $C E$ (see Fig. 13(iii)). Both of these edges already cross three other edges, so $\bar{g}$ ends in $\Gamma$, the face of $G^{\prime}$ adjacent 


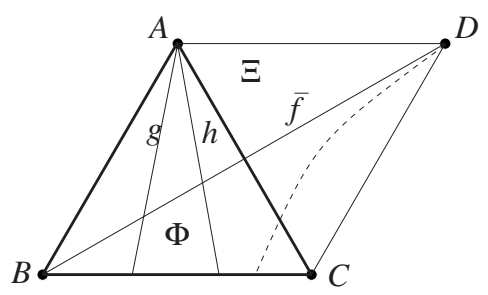

(i)

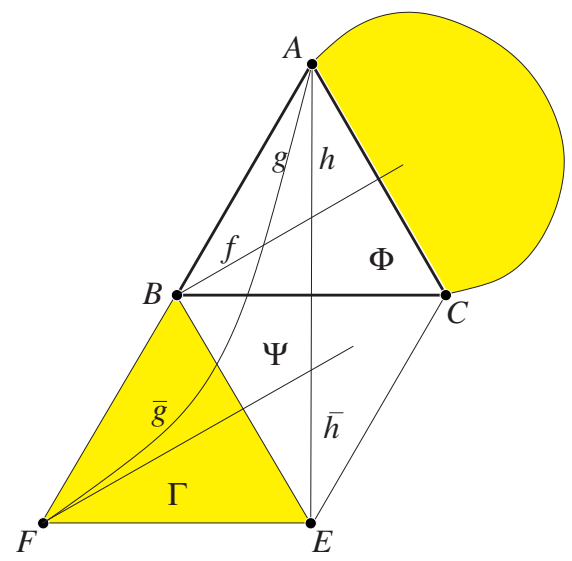

(ii)

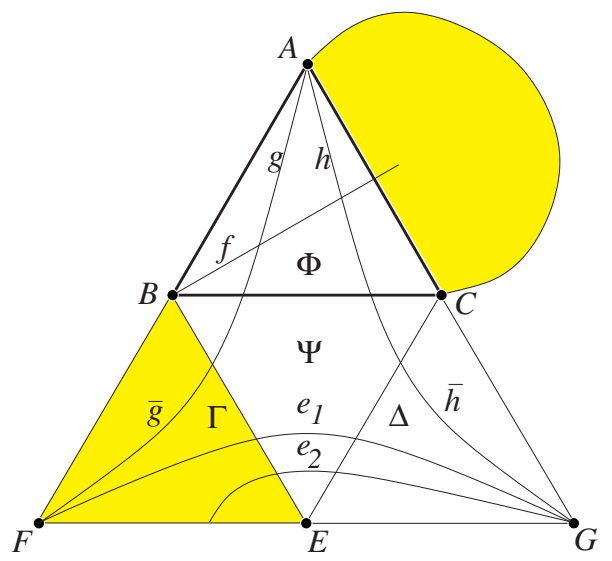

(iii)

Fig. 13. Proof of Lemma 2.3, Case 1. (A more detailed version of Fig. 4(b)-(d).)

to $\Psi$ along $B E$, and $\bar{h}$ ends in $\Delta$, the face of $G^{\prime}$ adjacent to $\Psi$ along $C E$. Both $\Gamma$ and $\Delta$ are triangles by assumption. Let $F$ and $G$ denote the vertices of $\Gamma$ and $\Delta$, where $g$ and $h$ end, respectively. As before, we easily conclude that $d_{\Gamma}(B)=d_{\Gamma}(E)=0$ and $d_{\Delta}(C)=d_{\Delta}(E)=0$. Since $\bar{h}$ is perfect, then $\Delta$ is a 3-triangle and $d_{\Delta}(G)=3$. Let $e_{1}$ and $e_{2}$ denote the extensions of the half-edges contributing to $d_{\Delta}(G)$ (other than $\bar{h}$ ). These edges exit $\Delta$ through $C E$ and enter $\Psi$. Neither of them can exit $\Psi$ through $B C$ or end in $B$, since $\bar{f}, \bar{g}$, and $\bar{h}$ already cross three other edges. Hence, $e_{1}$ and $e_{2}$ exit $\Psi$ through $B E$ and enter $\Gamma$. Now, there are only two possibilities: either $e_{1}$ and $e_{2}$ both exit $\Gamma$ through $F E$; or one of them ends in vertex $F$, while the other exits $\Gamma$ through $F E$ (see Fig. 13(iii)). In both cases $B E$ is crossed by three edges $\left(e_{1}, e_{2}, \bar{g}\right)$, and $d_{\Gamma}(F) \leq 2$. Therefore, $\Gamma$ is not a 3 -triangle, contradicting the assumption that $\bar{g}$ is perfect.

\section{Appendix 2. Proof of Claim A}

Recall that $\Psi$ is a simple face of $G^{\prime}$ with $|\Psi|=4$ and $|H(\Psi)|=6$. Next, we introduce some notation. Let $A, B, C$, and $D$ denote the vertices of $\Psi$, and let $d_{V}$ be the degree of $V \in\{A, B, C, D\}$ in $\Psi$, that is, the number of half-edges in $H(\Psi)$ incident to vertex 
$V$. Encode each half-edge by its type, consisting of the initial vertex and the side of $\Psi$ where it ends. So, for example, a half-edge of type $A(B C)$ connects vertex $A$ with the side $B C$. Finally, let $\Delta$ denote the maximum degree of all the vertices of $\Psi$.

Case 1: $\Delta=6$. Suppose that $d_{A}=\Delta$. Since at most three half-edges can exit $\Psi$ through the same side, there is only one possibility, depicted in Fig. 5(a).

Case 2: $\Delta=5$. Let $A$ be the vertex of degree 5. Three of the half-edges incident to $A$ exit through the same side, say $B C$, and two through the side $C D$. The remaining half-edge of $H(\Psi)$ cannot have its endpoint on $A B$ or on $B C$, and it cannot emanate from $B$. Therefore, it has to be of type $C(A D)$ (see Fig. 5(b)).

Case 3: $\Delta=4$. Let $d_{A}=\Delta$. There are two possibilities:

Case 3.1: Two of the half-edges incident to A exit $\Psi$ through side BC, while the other two exit through side $C D$. If there is a half-edge incident to $B$, it should exit through $C D$. However, then the remaining half-edge cannot be drawn: clearly, it cannot start at $C$ or $D$, and if it starts at $B$, then the two half-edges incident to $B$ have to be of type $B(C D)$, forcing at least four crossings on $C D$. Similarly, no half-edge can be incident to $D$. Therefore, the remaining two half-edges both emanate from $C$. By Lemma 2.1, they should exit $\Psi$ through different sides, giving Fig. 5(c).

Case 3.2: There are three half-edges in $H(\Psi)$ of type $A(C D)$ and one of type $A(B C)$. Then the remaining two half-edges cannot have their endpoints on $A D, C D$, or in $D$. So, they are both of type $C(A B)$ (see Fig. 5(d)).

Case 4: $\Delta=3$. Let $A$ be a vertex of degree 3. Again, there are two possibilities (up to symmetry).

Case 4.1: All three half-edges incident to $A$ are of the same type, say $A(B C)$. The remaining three half-edges of $H(\Psi)$ cannot have their endpoints on $A B$, on $B C$, or in $B$. Therefore, all of them are of type $C(A D)$, as shown in Fig. 5(e).

Case 4.2: Two half-edges incident to $A$ are of type $A(B C)$, while the remaining one is of type $A(C D)$. If there is a half-edge incident to $B$, it can only be of type $B(C D)$, Then, by Lemma 2.1, there are no more half-edges emanating from $B$. Moreover, no half-edge is incident to $C$; otherwise, any half-edge from $C$ would cross the existing half-edge of type $B(C D)$, whose extension already crosses three other edges. Similarly, at most one half-edge emanates from $D$ (extensions of the half-edges of type $A(B C)$ already cross two other edges). This contradicts $|H(\Psi)|=6$.

If there is a half-edge incident to $D$, it can only be of type $D(B C)$, and it has to be the unique half-edge of this type. The remaining two half-edges of $H(\Psi)$ must be incident to $C$. None of them can exit $\Psi$ through $A B$, so they are both of type $C(A D)$. However, then the extension of the existing half-edge of type $A(C D)$ crosses four other edges.

Therefore, we can assume that there are two half-edges of type $A(B C)$, one of type $A(C D)$, and the other three half-edges are incident to $C$. It is impossible that all three are of type $C(A D)$, since they would all cross the half-edge of type $A(C D)$. Moreover, by Lemma 2.1, at most one can be of type $C(A B)$. Therefore, one is of type $C(A B)$ and two are of type $C(A D)$, see Fig. 5(f).

Case 5: $\Delta=2$. First, suppose that for every vertex of degree 2 the two half-edges incident to it exit $\Psi$ through different sides. Also, assume that $d_{A}=2$, i.e., there is a half-edge of 
type $A(B C)$ and a half-edge of type $A(C D)$. If $B$ is of degree 2, then there is a half-edge of type $B(C D)$ and a half-edge of type $B(A D)$. Now, it is easy to see that at most one further half-edge can be added, either of type $C(A D)$ or of type $D(B C)$, contradicting $|H(\Psi)|=6$. If $C$ is of degree 2, for each of the four types, $A(B C), A(C D), C(A B)$, $C(A D)$, there is a unique half-edge of this type, whose extension is already crossed by two edges. Any additional half-edge emanating from either $B$ or $D$ would have to cross three of the above mentioned half-edges before reaching a side of $\Psi$. Hence, if $d_{C}=2$, then $d_{B}=d_{D}=0$, contradicting $|H(\Psi)|=6$.

Now, we can assume that there is a vertex (say, $A$ ) of degree 2 , such that both halfedges incident to it have the same type, say $A(C D)$. It follows from Lemma 2.1 that $d_{D} \leq 1$. If $d_{D}=0$, then $|H(\Psi)|=6$ implies $d_{B}=d_{C}=2$. Let us consider the two half-edges emanating from $B$. At most one of them is of type $B(C D)$. Furthermore, by Lemma 2.1, at most one of them is of type $B(A D)$. So, we have exactly one half-edge of type $B(C D)$ and one half-edge of type $B(A D)$. Any half-edge incident to $C$ would either have to cross three half-edges before reaching $A D$, or cross the existing half-edge of type $B(A D)$, whose extension already crosses three other edges. Therefore, we obtain $d_{C}=0$, a contradiction.

We are left with the case when there are two half-edges of type $A(C D)$, and $d_{D}=1$. If the half-edge incident to $D$ is of type $D(B C)$, then $d_{C}=0$, which, together with $d_{B} \leq 2$, gives $|H(\Psi)| \leq 5$, a contradiction. Therefore, the half-edge incident to $D$ has type $D(A B)$. In this case the half-edges incident to $B$ or $C$ cannot end on $A D$, so the possible types are $B(C D)$ and $C(A B)$. Since $C D$ is already crossed by two edges, there is at most one half-edge of type $B(C D)$. So there are two half-edges of type $C(A B)$, see Fig. 5(g). This concludes the proof of Claim A.

\section{References}

$\left[\mathrm{AC}^{+}\right]$M. Ajtai, V. Chvátal, M. Newborn, and E. Szemerédi, Crossing-free subgraphs, Annuals of Discrete Mathematics 12 (1982), 9-12.

$\left[\mathrm{CE}^{+}\right]$K. Clarkson, H. Edelsbrunner, L. Guibas, M. Sharir, and E. Welzl, Combinatorial complexity bounds for arrangements of curves and surfaces, Discrete \& Computational Geometry 5 (1990), 99-160.

$\left[\mathrm{CS}^{+}\right]$É. Czabarka, O. Sýkora, L. Székely, and I. Vrt'o, Biplanar crossing numbers, I: a survey of results and problems, in: More Sets, Graphs and Numbers, pp. 57-77, Bolyai Society Mathematical Studies, 15, Springer, Berlin, 2006.

[D] T. K. Dey, Improved bounds for planar $k$-sets and related problems, Discrete \& Computational Geometry 19 (1998), 373-382.

[ENR] G. Elekes, M. Nathanson, and I. Z. Ruzsa, Convexity and sumsets, Journal of Number Theory 83 (2000), 194-201.

[ErG] P. Erdôs and R. K. Guy, Crossing number problems, American Mathematical Monthly 80 (1973), $52-58$.

[KT] N. H. Katz and G. Tardos, A new entropy inequality for the Erdôs distance problem, in: Towards a Theory of Geometric Graphs (J. Pach, ed.), pp. 119-126, Contemporary Mathematics, 342, American Mathematical Society, Providence, RI, 2004.

[L] T. Leighton, Complexity Issues in VLSI, Foundations of Computing Series, MIT Press, Cambridge, MA, 1983.

[P1] J. Pach, Geometric graph theory, in: Surveys in Combinatorics, 1999 (J. D. Lamb and D. A. Preece, eds.), pp. 167-200, London Mathemetical Society Lecture Notes 267, Cambridge University Press, Cambridge, 1999.

[P2] J. Pach, Geometric graph theory, in: Handbook of Discrete and Computational Geometry, 2nd edn. (J. E. Goodman et al., eds.), pp. 219-238, CRC Press, Boca Raton, FL, 2004. 
[PS] J. Pach and M. Sharir, On the number of incidences between points and curves, Combinatorics, Probability, and Computing 7 (1998), 121-127.

[PST] J. Pach, J. Spencer, and G. Tóth, New bounds on crossing numbers, Discrete \& Computational Geometry 24 (2000), 623-644.

[PTa] J. Pach and G. Tardos, Isosceles triangles determined by a planar point set, Graphs and Combinatorics 18 (2002), 769-779.

[PTo1] J. Pach and G. Tóth, Graphs drawn with few crossings per edge, Combinatorica 17 (1997), 427-439.

[PTo2] J. Pach and G. Tóth, Which crossing number is it, anyway? Journal of Combinatorial Theory, Series B 80 (2000), 225-246.

[PSS] M. J. Pelsmajer, M. Schaefer, and D. Štefankovič, Odd crossing number is not crossing number, manuscript, 2005.

[ST] J. Solymosi and Cs. Tóth, Distinct distances in the plane, Discrete \& Computational Geometry 25 (2001), 629-634.

[STT] J. Solymosi, G. Tardos, and Cs. Tóth, The $k$ most frequent distances in the plane, Discrete \& Computational Geometry 28 (2002), 769-779.

[Sz] L. Székely, Crossing numbers and hard Erdôs problems in discrete geometry, Combinatorics, Probability, and Computing 6 (1997), 353-358.

[SzT] E. Szemerédi and W. T. Trotter, Extremal problems in discrete geometry, Combinatorica 3 (1983), 381-392.

Received July 29, 2004, and in revised form June 16, 2005, and July 25, 2005.

Online publication October 13, 2006. 